\title{
DETERMINAÇÃO DO PONTO DE AMOSTRAGEM PARA A QUANTIFICAÇÃO DE MACRONUTRIENTES EM Acacia mearnsii DE WILD.
}

\author{
Fábio Luiz Fleig Saidelles ${ }^{1}$, Marcos Vinicius Winckler Caldeira ${ }^{2}$, Mauro Valdir Schumacher ${ }^{3}$, \\ Rafaelo Balbinot ${ }^{4}$, Waldir Nagel Schirmer ${ }^{4}$ \\ ${ }^{1}$ Eng. Florestal, Dr. Fundação Estadual de Pesquisa Agropecuária (FEPAGRO), Santa Maria, RS, Brasil - fabio-saidelles@ fepagro.rs.gov.br \\ ${ }^{2}$ Eng. Florestal, Dr., Depto. de Eng. Florestal, UFES, Alegre, ES, Brasil - caldeiramv@ yahoo.com.br \\ ${ }^{3}$ Eng. Florestal, Dr., Depto. de Ciências Florestais,UFSM, Santa Maria, RS, Brasil - schumacher@ pesquisador.cnpq.br \\ ${ }^{4}$ Eng. Florestal, Dr., Depto. de Engenharia Ambiental, UNICENTRO, Irati, PR, Brasil - rbalbinot@ yahoo.com.br; wanasch@yahoo.com.br
}

Recebido para publicação: 21/07/2008 - Aceito para publicação: 10/06/2009

\begin{abstract}
Resumo
O objetivo deste estudo foi determinar o ponto ótimo de amostragem para a quantificação de nutrientes em árvores de Acacia mearnsii com quatro anos de idade. O trabalho foi realizado em um povoamento de acácia-negra localizado na cidade de Arroio dos Ratos/RS, em uma fazenda

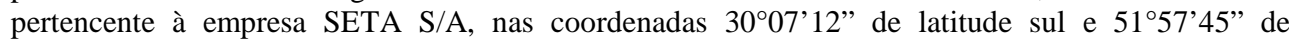
longitude, com altitude média de $90 \mathrm{~m}$. O primeiro passo foi a realização do inventário florestal no povoamento, onde foram derrubadas 21 árvores, distribuídas em 7 classes diamétricas, sendo determinados a biomassa e o teor de nutrientes dos componentes folha, galho vivo, galho morto, raiz, madeira e casca. Para os componentes madeira e casca, foram retiradas amostras ao longo de fuste nas posições de 1, 3, 10, 20, 30,40,50,60,70, 80 e 90\% da altura total das árvores. O ponto ótimo de amostragem para quantificação dos nutrientes (N, P, K, Ca, Mg e S) no fuste deve situar-se a $50 \%$ da altura total das árvores de Acacia mearnsii. É possível a estimativa do estoque de macronutrientes por amostras coletadas no DAP aos 4 anos de idade. Recomenda-se para isso a utilização de trado para não ser necessário derrubar as árvores amostradas.

Palavras-chave: Acácia-negra; ponto de amostragem; nutriente.
\end{abstract}

\begin{abstract}
Sampling point determination for macronutrients quantification on Acacia mearnsii De Wild. The objective of this study was to quantify the biomass and to determine the optimal sampling point for nutrients quantification in four year old Acacia mearnsii trees. It was conducted in black wattle stand, located in Arroio dos Ratos city in a farm belonging to SETA S/A, having as coordinates $30^{\circ} 07^{\prime} 12^{\prime \prime}$ of south latitude and $51^{\circ} 57^{\prime \prime} 45^{\prime \prime}$ of longitude, with $90 \mathrm{~m}$ of average altitude. The first step was the forest inventory, where 30 trees were felled, distributed in 7 diametric classes, to cover the stand heterogeneity, then the biomass and the nutrients amount in the leaves, live branches, dead branches, roots, wood and bark were determined. Samples of wood and bark were taken along the stem in the positions of: 1, 3, 10, 20, 30, 40, 50, 60, 70, 80 and $90 \%$ of the total height. The optimal sampling point for nutrients $(\mathrm{N}, \mathrm{P}, \mathrm{K}, \mathrm{Ca}, \mathrm{Mg}$, and $\mathrm{S}$ ) quantification along the stem should be 50\% from the total height in Acacia mearnsii trees. It's possible to estimate the stock of macronutrients by samples collected at DBH at 4 years of age. For this it's recommended to use the borer, for not to be necessary to fell the sampled trees.

Keywords: Black wattle; sampling point; nutrient.
\end{abstract}

\section{INTRODUÇÃO}

Acacia mearnsii De Wild., também conhecida por acácia-negra, apresentava, em 2000, segundo o Inventário Florestal Contínuo do RS (2005), uma área plantada no estado estimada em $96,40 \mathrm{~km}^{2}$, ou $0,03 \%$. Apesar da área de cobertura, a espécie é considerada uma das principais espécies reflorestadas em muitos municípios. 
A acacicultura apresenta uma série de vantagens socioeconômicas. Atividade ligada principalmente aos pequenos e médios produtores, cuja mão de obra é quase que exclusivamente familiar, o plantio da acácia-negra beneficia hoje mais de 40 mil famílias na região Sul do Brasil. Os aproveitamentos múltiplos, aliados à boa remuneração, fazem dessa cultura uma das melhores opções de investimento no setor primário. Além do rápido crescimento e de sua fácil propagação, a acácia-negra tem grandes vantagens perante as demais espécies florestais, devido ao seu aproveitamento integral de casca e madeira. Da casca, extrai-se o tanino, que é utilizado no curtimento de couro e peles. A madeira é matéria-prima de primeira qualidade para a fabricação de papel e celulose. Além disso, o carvão vegetal de acácia-negra é muito aceito no mercado, em virtude da sua produtividade, economia e isenção de odores (SETA, 2005).

As florestas de acácia-negra apresentam estreita relação com os pequenos produtores nas regiões onde são cultivadas, o que faz com que elas sejam cada vez mais consideradas a "salvação da lavoura". Cada vez mais sua colheita tem se tornado mais precoce, com redução da rotação, em busca de um retorno econômico mais rápido. Em muitos casos é colhida com quatro anos. Esse fato, associado à retirada da madeira e da casca no processo da colheita, pode ser considerado um problema quanto à exportação de nutrientes do sítio. O conhecimento do estoque de nutrientes tanto no fuste quanto nos demais componentes das árvores é de fundamental importância para um monitoramento constante dos nutrientes exportados, para o qual se deve conhecer o ponto para retirada das amostras ao longo do fuste.

Sabe-se que com o conhecimento da biomassa pode-se ter uma noção da quantidade de nutrientes armazenados nas espécies arbóreas e/ou de ecossistemas florestais, o que se faz necessário para aumentar a eficiência no manejo dos povoamentos e estabelecer um balanço nutricional adequado.

No entanto, a utilização de equações de regressão para estimar a biomassa e a quantidade de nutrientes dos componentes em povoamentos futuros permite, entre outros fatos, a utilização de métodos não-destrutivos, principalmente quando se trata do sistema radicular, que é de difícil amostragem.

Nesse sentido, o conhecimento dos teores dos nutrientes ao longo do fuste das árvores é importante para que se possa determinar um ponto exato para a retirada das amostras, o que facilitará a determinação das quantidades de nutrientes armazenados na madeira e na casca.

Portanto, o presente trabalho, realizado em povoamentos de Acacia mearnsii De Wild. com quatro anos de idade estabelecido no Rio Grande do Sul, tem como objetivos determinar a altura para amostragem dos componentes madeira e casca das árvores para a determinação dos macronutrientes.

\section{MATERIAL E MÉTODOS}

\section{Características da área de estudo}

O presente trabalho foi realizado em um povoamento de acácia-negra (Acacia mearnsii) com quatro anos de idade, implantado na Fazenda Chagastelles, pertencente à empresa Florestal Agroseta S.A., distante aproximadamente $4 \mathrm{~km}$ da cidade de Arroio dos Ratos/RS. Distando cerca de $60 \mathrm{~km}$ de Porto Alegre, o município de Arroio dos Ratos localiza-se na Depressão Central do Estado do Rio Grande Sul, na microrregião do Vale do Jacuí, limitada ao norte pela encosta do planalto basáltico e ao sul pelo escudo rio-grandense, situada entre as coordenadas geográficas: latitude $30^{\circ} 07^{\prime} 12^{\prime}$ ' sul e longitude $51^{\circ} 57^{\prime} 45^{\prime \prime}$ oeste de Greenwich, com altitude média de 90 metros.

Segundo Koeppen, o clima da região é do tipo Cfa, subtropical (MORENO, 1961). A temperatura média do mês de janeiro fica em torno de $24{ }^{\circ} \mathrm{C}$, a do mês de julho é $13{ }^{\circ} \mathrm{C}$ e a temperatura média anual fica entre $18-19^{\circ} \mathrm{C}$, sendo que a temperatura média das máximas no ano é de $24^{\circ} \mathrm{C}$ e a das mínimas no ano é de $14^{\circ} \mathrm{C}$. A precipitação pluvial no ano varia de 1200 a $1600 \mathrm{~mm}$ e é bem distribuída, ocorrendo de 100 a 120 dias de chuva no ano. A região de Arroio dos Ratos possui, de maio a agosto, 600 horas de frio, abaixo de $10{ }^{\circ} \mathrm{C}$, e 200 horas de frio de maio a agosto, abaixo de $7{ }^{\circ} \mathrm{C}$. A umidade relativa do ar média, radiação solar média e insolação anual total variam, respectivamente, de 75 a $80 \%$, de 325 a $375 \mathrm{cal} . \mathrm{cm}^{-2} \cdot \mathrm{dia}^{-1} \mathrm{e}$ de 2200 a 2600 horas (INSTITUTO DE PESQUISAS AGRONÔMICAS, 1989).

O solo da área de estudo (Tabela 1), segundo a EMBRAPA (1999), é classificado como Argissolo Vermelho Distrófico típico, localizado em relevo suave ondulado. 
Tabela 1. Análise química do solo plantado com Acacia mearnsii De Wild., com quatro anos de idade, Arroio dos Ratos, RS/Brasil.

Table 1. Chemical analysis of soil planted with Acacia mearnsii with four years old, Arroio dos Ratos, RS/Brazil.

\begin{tabular}{|c|c|c|c|c|c|c|c|c|c|c|c|c|c|c|}
\hline \multirow{2}{*}{$\begin{array}{l}\text { Prof. } \\
\text { cm }\end{array}$} & \multirow{2}{*}{$\begin{array}{l}\text { Argila } \\
\text { g.kg-1 }^{-1}\end{array}$} & \multirow{2}{*}{$\begin{array}{c}\mathrm{pH} \\
\mathrm{H}_{2} \mathrm{O}\end{array}$} & M.O & $\mathbf{m}$ & $\mathbf{V}$ & \multirow{2}{*}{\multicolumn{2}{|c|}{$\begin{array}{l}\mathbf{P} \quad \mathbf{K} \\
\text { mg.L }^{-1}\end{array}$}} & \multirow{2}{*}{\multicolumn{3}{|c|}{$\begin{array}{lll}\text { Ca } & \text { Mg } & \text { CTC } \\
\text { cmol }_{c} \cdot \mathrm{L}^{-1}\end{array}$}} & \multirow[t]{2}{*}{$\mathbf{C u}$} & \multirow{2}{*}{\multicolumn{2}{|c|}{$\underset{\text { mg.L }}{\mathbf{Z n}} \underset{\mathrm{Fe}}{\mathrm{Fe}}$}} & \multirow[t]{2}{*}{ Mn } \\
\hline & & & \multicolumn{3}{|c|}{$\%$} & & & & & & & & & \\
\hline $0-10$ & 310 & 4,4 & 2,5 & 27 & 44 & 4,0 & 86 & 2,0 & 0,5 & 4,8 & 1,8 & 1,6 & 123 & 90 \\
\hline 10-20 & 350 & 4,3 & 2,0 & 27 & 41 & 3,2 & 112 & 1,5 & 0,5 & 3,9 & 1,9 & 1,3 & 133 & 76 \\
\hline $20-30$ & 440 & 4,4 & 1,8 & 30 & 39 & 3,2 & 114 & 2,2 & 0,8 & 4,7 & 2,3 & 0,8 & 64 & 38 \\
\hline $30-40$ & 460 & 4,4 & 1,8 & 41 & 35 & 3,2 & 78 & 2,1 & 0,9 & 4,9 & 2,2 & 0,5 & 54 & 6 \\
\hline $40-50$ & 430 & 4,4 & 1,7 & 33 & 40 & 3,2 & 78 & 1,8 & 0,8 & 4,7 & 2,0 & 0,4 & 51 & 6 \\
\hline $50-60$ & 560 & 4,4 & 1,6 & 35 & 40 & 2,5 & 90 & 1,9 & 0,9 & 5,0 & 1,9 & 0,4 & 45 & 1 \\
\hline
\end{tabular}

\section{Implantação do povoamento}

O plantio foi realizado em setembro de 1997, numa área de 2.564 ha, com espaçamento de $1,30 \mathrm{~m} \times 3,0 \mathrm{~m}$, tendo como preparo de solo a subsolagem com uma haste, alcançando a profundidade de $45 \mathrm{~cm}$, e gradagem na linha de plantio. O plantio foi realizado com semente, utilizando-se plantadeira hidráulica de acácia-negra (PHA). Em cada cova foram colocadas oito sementes. Simultaneamente ao plantio, foi realizada a adubação, utilizando-se 40 gramas por cova de NPK (02-32-06). Outra etapa foi o replantio, realizado aproximadamente 30 dias após a implantação do povoamento.

Em relação aos tratos culturais, foi aplicado herbicida seletivo pré-emergente (Gamit $360 \mathrm{cs}$ ), na dose de $400 \mathrm{ml} \cdot \mathrm{ha}^{-1}$, e em maio de 1998 foi realizado desbaste e limpeza da área, utilizando-se foice manual. Foi realizado ainda o combate à formiga-cortadeira e, mais tarde, o controle do serrador (Oncideres inpluviata).

\section{Inventário florestal da área amostral}

Inicialmente, foi escolhida uma área homogênea quanto às condições de sítio, onde foram demarcadas 7 parcelas de formato retangular de $20 \times 30 \mathrm{~m}\left(600 \mathrm{~m}^{2}\right)$. Em cada uma das parcelas foram medidos os diâmetros à altura do peito (DAP) de todas as árvores, com suta, e a altura (h) de 10\% das alturas das mesmas, com aparelho Vertex, conforme a metodologia proposta por Caldeira et al. (2002).

As alturas das árvores que não foram medidas foram estimadas com uma relação hipsométrica, que apresentou os melhores parâmetros de ajuste para a região e para a espécie, na época da realização da pesquisa (Tabela 2).

Tabela 2. Equação de regressão testada para estimar as alturas de Acacia mearnsii com quatro anos de idade, Arroio dos Ratos, RS/Brasil.

Table 2. Equations used for regression analysis to estimate heights for four years old Acacia mearnsii, Arroio dos Ratos, RS/Brazil.

\begin{tabular}{lcccc}
\hline \multirow{2}{*}{ Modelo } & \multicolumn{2}{c}{ Coeficientes } & \multirow{2}{*}{$\mathbf{R}_{\mathrm{aj}}{ }^{2}$} & \multirow{2}{*}{ Syx $(\boldsymbol{\%})$} \\
\cline { 2 - 3 } & $\mathbf{b}_{\mathbf{0}}$ & $\mathbf{b}_{\mathbf{1}}$ & & \\
\hline $\log \mathrm{h}=\mathrm{b}_{0}+\mathrm{b}_{1} *$ 1/DAP & 1,378574 & $-2,079563$ & 0,92 & 3,0 \\
\hline H: altura $(\mathrm{m}) ; \log :$ logaritmo de base 10; DAP: diâmetro à altura do peito $(\mathrm{cm}) ; \mathrm{R}_{\mathrm{aj}}^{2}$ : coeficiente de determinação; $\mathrm{S}_{\mathrm{yx}}(\%)$ : erro padrão.
\end{tabular}

O volume total com e sem casca foi estimado com base nas equações propostas por Schneider; Hosokawa (1978), a partir dos dados de DAP e altura do povoamento (Tabela 3).

Tabela 3. Equações utilizadas para cálculo do volume total com e sem casca para Acacia mearnsii De Wild. com quatro anos de idade, Arroio dos Ratos, RS/Brasil.

Table 3. Equations utilized for four years old Acacia mearnsii De Wild. total volume calculation with and without bark, Arroio dos Ratos, RS/Brazil.

\begin{tabular}{lcc}
\hline \multirow{2}{*}{ Modelo } & \multicolumn{2}{c}{ Coeficientes } \\
\cline { 2 - 3 } & $\mathbf{b}_{\mathbf{0}}$ & $\mathbf{b}_{\mathbf{1}}$ \\
\hline $\log \mathrm{vcc}=\mathrm{b}_{0}+\mathrm{b}_{1} * \log \left(\mathrm{DAP}^{2} \mathrm{~h}\right)$ & $-4,20076$ & 0,9494568 \\
$\log \mathrm{vsc}=\mathrm{b}_{0}+\mathrm{b}_{1}{ }^{*} \log \left(\mathrm{DAP}^{2} \mathrm{~h}\right)$ & $-4,29654$ & 0,9562276 \\
\hline
\end{tabular}

H: altura $(\mathrm{m})$; log: logaritmo de base 10; DAP; diâmetro à altura do peito $(\mathrm{cm})$; vsc: volume total sem casca $\left(\mathrm{m}^{3}\right)$; vcc: volume total com casca $\left(\mathrm{m}^{3}\right) ; \mathrm{R}_{\mathrm{aj}}{ }^{2}(97,11)$; Syx $(0,002 \%)$. 
A partir dos dados do inventário das parcelas, o povoamento foi caracterizado, agrupando-se as árvores em classes de diâmetro, de modo a abranger as variações ocorridas no povoamento. Para tanto, determinou-se o número de classes, por meio da fórmula de Sturges descrita por Finger (1992), a qual é expressa por: $\mathrm{K}=1+3,3 * \log \mathrm{n}$, onde $\mathrm{n}=$ número de observações e $\mathrm{K}=$ número de classes. Também foi determinado o intervalo de classes, com base na fórmula: $h=H / K$, onde $H=$ amplitude total (DAP máx DAP $\min ), \mathrm{K}=$ número de classes e $\mathrm{h}=$ intervalo de classe $(\mathrm{cm})$. Na figura 1 , tem-se a distribuição do número de árvores por hectare. Observa-se que a maioria das árvores encontra-se nas classes intermediárias.

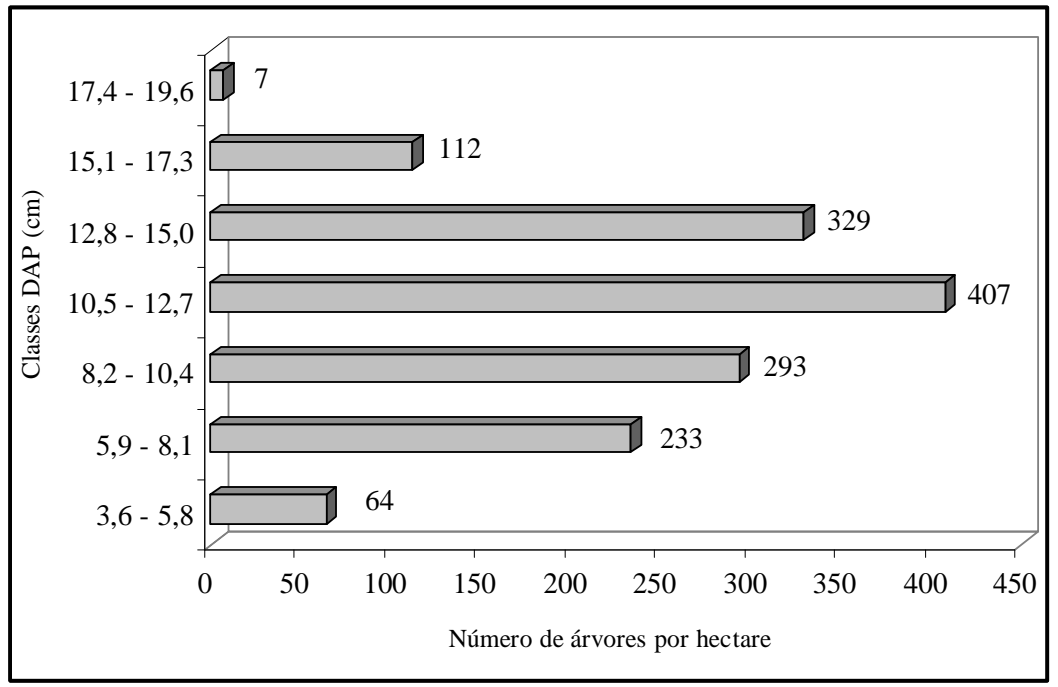

Figura 1. Número de árvores de Acacia mearnsii por hectare, distribuídas nos intervalos de classes de DAP com quatro anos de idade, Arroio dos Ratos, RS/Brasil.

Figure 1. Number of trees per hectare of four years old Acacia mearnsii distributed in BHD classes intervals, Arroio dos Ratos, RS/Brazil.

\section{Biomassa acima e abaixo do solo}

Após a determinação da distribuição diamétrica e sua divisão em classes de diâmetro, foram selecionadas três árvores em cada uma delas, de modo que a maior amplitude dos dados fosse contemplada. O DAP e a altura total foram medidos e em seguida os galhos foram separados do tronco e classificados em vivos (verdes) e mortos (secos). Dos galhos verdes foram separadas todas as folhas. O tronco foi descascado, sendo separado em dois componentes: madeira e casca.

Ainda no campo, os componentes (madeira, casca, folhas, galhos vivos e galhos mortos) tiveram seu peso úmido determinado, mediante pesagem com utilização de uma balança de gancho. Logo após, foram retiradas amostras de todos os componentes das árvores e pesadas em balança de precisão $(0,01 \mathrm{~g})$. Para a madeira e casca, foram retiradas amostras nas posições de 1, 3, 10, 20, 30, 40, 50, 60, 70, 80 e 90\% da altura total das árvores.

Com o peso úmido determinado no campo, as amostras foram devidamente identificadas e encaminhadas para o Laboratório de Ecologia Florestal da Universidade Federal de Santa Maria, onde foram postas em estufa de circulação de ar, à temperatura de $65^{\circ} \mathrm{C}$, até atingirem peso constante, para obtenção do peso seco das amostras (CALDEIRA, 1998).

Com o peso seco e úmido das amostras, estabeleceu-se a relação peso seco/úmido. Com o peso úmido total de cada componente, foram calculados os pesos secos de cada componente de árvore e, com isso, procedeu-se a determinação da biomassa seca $(\mathrm{kg})$.

As 21 árvores amostradas tiveram suas raízes escavadas com auxílio de um trator. Por meio do uso de bomba de pressão, foram submetidas a lavagem, na qual o solo aderido às raízes foi totalmente removido e todas as raízes com diâmetro acima de $0,50 \mathrm{~cm}$ foram coletadas (BARICHELLO, 2003; HOPPE, 2003; SAIDELLES, 2005). As amostras da raiz foram retiradas nesse momento, com a 
determinação do seu peso úmido, sendo posteriormente levadas ao laboratório, onde foi realizado o mesmo procedimento adotado para as outras amostras.

\section{Teor de macronutrientes nos componentes das árvores}

Para a determinação do teor de nutrientes nos diferentes componentes das árvores de acácianegra, foram utilizadas as amostras anteriormente coletadas para a determinação da biomassa de folhas, galho vivo, galho morto, madeira, casca e raiz. No laboratório de Ecologia Florestal, as amostras foram secas, moídas e submetidas a análises químicas para obtenção dos teores dos nutrientes.

Os macronutrientes determinados foram nitrogênio $(\mathrm{N})$, fósforo $(\mathrm{P})$, potássio $(\mathrm{K})$, cálcio $(\mathrm{Ca})$, magnésio $(\mathrm{Mg})$ e enxofre (S), com base na metodologia descrita por Tedesco et al. (1995) para análises de tecidos vegetais.

\section{Relação entre o DAP e o teor de macronutrientes}

Com os dados dos teores de macronutrientes $\left(\mathrm{g} \cdot \mathrm{kg}^{-1}\right)$ obtidos na análise dos tecidos pelo produto com a biomassa de cada componente $(\mathrm{kg})$, estimou-se a quantidade de macronutrientes dos componentes $\left(\mathrm{g}\right.$.árvore $\left.{ }^{-1}\right)$. Com a variável dendrométrica (DAP) das respectivas árvores amostradas, foi usada uma equação ( $\log \mathrm{y}=\mathrm{b}_{0}+\mathrm{b}_{1} \log \mathrm{DAP}$ ) para cada macronutriente em cada componente das árvores. Para cada equação, foram utilizados parâmetros estatísticos para averiguar se a equação apresentava um bom ajuste aos dados, sendo eles o coeficiente de determinação ajustado $\left(\mathrm{R}_{\mathrm{aj}}{ }_{\mathrm{aj}}\right.$ ) e o erro padrão da estimativa em percentagem $\left(\mathrm{S}_{\mathrm{yx}} \%\right)$. Só então as equações foram utilizadas para estimar os conteúdos dos nutrientes dos componentes das árvores de Acacia mearnsii do restante da parcela.

\section{Quantidade de macronutrientes nos componentes da biomassa por hectare}

A quantidade de macronutrientes por hectare $\left(\mathrm{kg} \cdot \mathrm{ha}^{-1}\right)$ foi determinada com base na média do teor de cada macronutriente $\left(\mathrm{g} \cdot \mathrm{kg}^{-1}\right)$ dos componentes das árvores multiplicada pela biomassa $\left(\mathrm{mg} \cdot \mathrm{ha}^{-1}\right)$ dos respectivos componentes, por classe diamétrica. O total por hectare foi obtido pela somatória dos valores por classe diamétrica.

\section{Teor de macronutrientes ao longo do fuste para madeira e casca}

Foram coletadas amostras ao longo do fuste nas posições a 1, 3, 10, 20,..., 80 e $90 \%$ da altura total das árvores. As amostras foram secas, moídas e analisadas para determinação dos teores dos macronutrientes nesses compartimentos.

\section{Teor representativo}

$\mathrm{O}$ teor de macronutriente ao longo do fuste (madeira e casca) é chamado de teor representativo e serve para a determinação do teor de macronutrientes no fuste (HOPPE, 2003).

Para o cálculo do teor representativo, que nada mais é do que o teor de macronutriente ponderado em função da biomassa, utilizou-se a massa de macronutriente de cada árvore, obtida pela multiplicação do teor de macronutriente encontrado nas posições amostradas ao longo do fuste pelas respectivas biomassas dos componentes (madeira e casca), expressa em grama (g) para os macronutrientes. Com o quociente da massa total de macronutriente e a biomassa total acumuladas nos componentes (madeira e casca) das 21 árvores amostradas, obteve-se o teor representativo.

\footnotetext{
Altura representativa

A expressão altura representativa expressa a altura no fuste da qual pode ser retirada uma amostra que represente a quantidade de macronutrientes no fuste para os componentes (madeira e casca). Para determinar a altura representativa da amostragem para a quantificação dos macronutrientes no fuste, para madeira e casca, deve-se, em primeiro lugar, observar qual o macronutriente que apresentou variação ao longo do fuste e, depois, usar o teor representativo para determinar a altura ideal da amostragem.

\section{Análise estatística}

Com objetivo de verificar se existem diferenças entre o teor dos macronutrientes nos componentes (madeira e casca) nas diferentes alturas amostradas, o delineamento utilizado foi
} 
inteiramente casualizado. Através da ANOVA e do teste de Tukey ao nível de 5\%, é possível verificar se existe diferença significativa entre os teores encontrados ao longo do fuste.

\section{RESULTADOS E DISCUSSÃO}

\section{Teor de macronutrientes nos componentes arbóreos}

$\mathrm{Na}$ tabela 4, constam os teores dos macronutrientes encontrados nos diferentes componentes das árvores de acácia-negra. Os maiores teores de todos os macronutrientes analisados foram encontrados nas folhas, exceto $\mathrm{Ca}$, que teve a maior concentração na casca e os menores teores na madeira e nas raízes.

Tabela 4. Teores médios de macronutrientes $\left(\mathrm{g}^{\mathrm{kg}} \mathrm{kg}^{-1}\right)$ nos diferentes componentes das árvores de Acacia mearnsii De Wild. com quatro anos de idade, Arroio dos Ratos, RS/Brasil.

Table 4. Macronutrients average levels $\left(\mathrm{g}_{\mathrm{kg}} \mathrm{kg}^{-1}\right.$ ) in different components of four years old Acacia mearnsii De Wild. trees, Arroio dos Ratos, RS/Brazil.

\begin{tabular}{lcccccc}
\hline \multirow{2}{*}{ Componente } & $\mathbf{N}$ & $\mathbf{P}$ & $\mathbf{K}$ & $\mathbf{C a}$ & $\mathbf{M g}$ & $\mathbf{S}$ \\
\cline { 2 - 7 } & & \multicolumn{9}{c}{$\mathrm{g}_{\mathrm{kg}}{ }^{-1}$} & & & \\
\hline Folha & 28,52 & 1,17 & 9,59 & 5,81 & 2,45 & 0,77 \\
Raiz & 5,90 & 0,22 & 2,67 & 2,80 & 0,52 & 0,34 \\
Madeira & 1,58 & 0,09 & 1,30 & 2,87 & 0,45 & 0,18 \\
Casca & 10,62 & 0,37 & 5,95 & 7,78 & 1,82 & 0,27 \\
Galho morto & 8,73 & 0,28 & 2,68 & 5,70 & 2,00 & 0,42 \\
Galho vivo & 10,13 & 0,61 & 8,18 & 4,31 & 1,47 & 0,42 \\
\hline
\end{tabular}

No que se refere aos teores de Ca na madeira e nas raízes, a tabela 4 mostra que entre os dois componentes a diferença é de $0,07 \mathrm{~g}$ em termos relativos. No entanto, quando se trata do acúmulo (quantidade) de Ca nesses dois componentes, a madeira é o componente arbóreo que estoca a maior quantidade (Tabela 6), em função de produzir mais biomassa.

Segundo Caldeira (1998), as diferentes concentrações de nutrientes nas diferentes espécies podem ser atribuídas às características genéticas de cada espécie e também às condições edáficas nas quais as espécies se desenvolvem. Em seu trabalho com diferentes procedências de acácia-negra, o autor observou que, entre as procedências utilizadas, não foi verificada diferença quanto à concentração dos nutrientes nos diferentes componentes da biomassa aérea. Em todas as procedências, foram encontrados, nas folhas, os maiores teores de $\mathrm{N}, \mathrm{P}, \mathrm{K}, \mathrm{Mg}$ e $\mathrm{S}$.

A concentração de nutrientes das folhas das árvores é influenciada por diversos fatores, como a idade das árvores, a estação do ano (VAN DER DRIESSCHE, 1984; BELLOTE, 1990), as condições do sítio e a posição em que as folhas estão dispostas na copa (VAN DER DRIESSCHE, 1984).

Do ponto de vista nutricional, Poggiani et al. (1984) comentaram que os valores mais elevados são encontrados nas folhas. No entanto, em alguns casos, os maiores teores de potássio, fósforo e principalmente cálcio podem ser observados na casca.

Para Gonçalves et al. (2000), os maiores teores de nutrientes são encontrados nas partes das plantas mais ativas metabolicamente, como as folhas, e as menores concentrações de micronutrientes são encontradas na madeira. Contudo, para a maioria dos nutrientes, é na madeira que se encontram os maiores conteúdos desses nutrientes, simplesmente devido a sua maior massa seca.

Em trabalho realizado em um povoamento de acácia-negra com 6 anos estabelecido no Rio Grande do Sul, Calil (2003) observou que os maiores teores de nutrientes foram encontrados nas folhas, enquanto que as menores concentrações foram encontradas na madeira, com exceção do fósforo, que se apresentou em menor concentração nas raízes. Essa autora atribuiu os altos teores de nitrogênio nas folhas à capacidade dessa espécie de fixar o nitrogênio atmosférico pela simbiose com bactérias do gênero Rhizobium (FRANCO et al., 1994). Os altos teores de Mg encontrados nas folhas são devidos ao fato desse macronutriente fazer parte da clorofila $\mathrm{a}$ e $\mathrm{b}$ presentes nas folhas, nas quais encontra-se a maioria das células vivas, proporcionando o acúmulo das maiores quantidades de nutrientes para os vários processos que ocorrem nesse órgão (KRAMER; KOZLOWSKI, 1972). Resultados semelhantes foram encontrados por Barichello (2003) e Caldeira (2003). 
Em seu estudo com Acacia mearnsii com 2,4 anos, Caldeira (1998) verificou que, apesar da madeira apresentar maior contribuição em relação às folhas, na biomassa acima do solo, as folhas apresentaram maior quantidade de nutriente que a madeira.

Hoppe (2003) verificou que a madeira é o componente das árvores de Platanus x acerifolia que apresentou os menores teores de todos os macronutrientes. As folhas apresentaram os maiores teores de $\mathrm{N}$ e $\mathrm{Mg}$, a casca apresentou os maiores teores de $\mathrm{Ca}$ e $\mathrm{S}$, enquanto que os frutos, os maiores teores de $\mathrm{P}$ e $\mathrm{K}$.

Estudo realizado por Weber (2004) observou que os teores médios de N, P, K, Mg e S em g.kg-1 , para Cryptomeria japonica com 28 anos, foram encontrados nas folhas, enquanto que os maiores teores de $\mathrm{Ca}$ foram encontrados na casca. Para Freitas (2000), trabalhando com Eucalyptus sp., os maiores teores de N e K foram encontrados nas folhas, enquanto que, para o $\mathrm{P}, \mathrm{Ca}$ e $\mathrm{Mg}$, os maiores valores localizaram-se na casca.

\section{Estimativas da quantidade de macronutrientes nos componentes arbóreos}

As equações ajustadas com os dados das árvores amostradas, com o objetivo de estimar os macronutrientes por árvore, com base no DAP das árvores, estão na tabela 5. A variável DAP teve um bom ajuste dos dados, expresso pelo alto coeficiente de determinação e pelo baixo erro padrão apresentado pela maioria das equações. Desse modo, o DAP é indicado como entrada para estimar macronutrientes da árvore e, principalmente, por apresentar facilidade de obtenção a campo.

\section{Quantidade de macronutrientes nos componentes da biomassa por hectare}

A tabela 6 mostra o estoque acumulado de macronutrientes nos diferentes componentes das árvores de acácia-negra, por hectare. Pode-se verificar que a madeira é o componente das árvores que estoca a maior quantidade de macronutrientes, com exceção do nitrogênio, que apresentou as maiores quantidades na casca. A maior quantidade dos macronutrientes na madeira foi devido a ela apresentar a maior contribuição da biomassa por hectare $(64 \%)$. Resultados semelhantes também foram observados por Caldeira (1998); Pereira et al. (2000) e Barichello (2003) com Acacia mearnsii.

Cabe ressaltar que a quantidade de nutrientes, segundo Caldeira (1998), é consequência de suas concentrações e da produção de biomassa do povoamento. Nesse sentido, Calil (2003), para acácia-negra com 6 anos, afirma que a madeira é o componente que apresenta os maiores conteúdos de macronutrientes.

Os resultados encontrados neste trabalho (Tabela 6) vêm confirmar o que Weber (2004) constatou em povoamento de Cryptomeria japonica com 28 anos, que a madeira, apesar de ter apresentado a maior constituição (67\%) da biomassa arbórea acima do solo, representou $29 \%$ da quantidade total de $\mathrm{N}\left(\mathrm{kg} \cdot \mathrm{ha}^{-1}\right), 45 \%$ do P, $60 \%$ do K, $29 \%$ do Ca, $43 \%$ do Mg e $49 \%$ do S. Já nas folhas, que apresentaram apenas 5\% da biomassa total, os macronutrientes representam, do total dos elementos, $31 \%$ do N, $23 \%$ do P, $17 \%$ do K, $10 \%$ do $\mathrm{Ca}, 26 \%$ do $\mathrm{Mg}$ e $24 \%$ do S.

\section{Ponto de amostragem para a quantificação dos macronutrientes no fuste}

Segundo Hoppe (2003), o conhecimento dos teores dos nutrientes ao longo do fuste das árvores é importante porque se pode determinar um ponto exato para a tomada de amostras, o que facilitará a determinação dos teores de nutrientes armazenados na casca e na madeira das árvores. Embora seja uma informação valiosa, o conhecimento da concentração dos nutrientes ao longo do fuste, tanto da casca quanto da madeira, ainda é pouco conhecido, devido à inexistência de pesquisa sobre esse tema.

Observa-se, na tabela 7, que os teores médios dos macronutrientes não apresentaram diferenças significativas nas diferentes alturas de amostragem ao longo de fuste para acácia-negra, com exceção do $\mathrm{N}$ e do $\mathrm{K}$ na madeira e do $\mathrm{N}$ e do $\mathrm{P}$ na casca. Mas não se pode deixar de notar que, tanto para a madeira quanto para a casca, quanto mais alta a posição no fuste, maior é o teor do $\mathrm{N}$, sendo isso associado à mobilidade desse elemento. Note-se que P e K também são considerados móveis, portanto a mobilidade é responsável apenas em parte.

Hoppe (2003) observou para a madeira e a casca do Platanus $x$ acerifolia que os teores de macronutrientes apresentavam distribuição variada: $\mathrm{N}, \mathrm{P}$ e K com maiores teores nas partes mais altas das árvores, confirmando a condição de móvel dentro da planta. Já quanto ao Ca e ao S, para ambos os componentes, os maiores teores foram encontrados na parte basal das árvores. $\mathrm{O}$ teor $\mathrm{de} \mathrm{Mg}$, ao longo do fuste, para a madeira, teve seu maior valor localizado na base, enquanto que para a casca, os maiores teores foram encontrados nas partes mais altas. 
Tabela 5. Equações de regressão utilizadas para estimar o estoque de macronutrientes (g/árvore) contidos nos diferentes componentes das árvores de Acacia mearnsii De Wild. com quatro anos de idade, Arroio dos Ratos, RS/Brasil.

Table 5. Regression equations utilized to estimate macronutrients stock ( $\mathrm{g} /$ trees) in different components of for four years old Acacia mearnsii De Wild. trees, Arroio dos Ratos, RS/Brazil.

\begin{tabular}{|c|c|c|c|}
\hline Nutriente & Equação & $\mathbf{R}_{\text {aj. }}^{2}$ & $\mathrm{~S}_{\mathrm{yx}}(\%)$ \\
\hline \multicolumn{4}{|c|}{ Raiz } \\
\hline $\mathrm{N}$ & $\log y=-1,33765+2,639628 * \log \mathrm{DAP}$ & 0,92 & 11,8 \\
\hline $\mathrm{P}$ & $\log y=-2,79893+2,682343 * \log \mathrm{DAP}$ & 0,94 & 15,7 \\
\hline $\mathrm{K}$ & $\log y=-1,60904+2,572228 * \log$ DAP & 0,92 & 16,0 \\
\hline $\mathrm{Ca}$ & $\log y=-1,42694+2,396759 * \log \mathrm{DAP}$ & 0,88 & 17,9 \\
\hline $\mathrm{Mg}$ & $\log y=-2,36918+2,636113 * \log \mathrm{DAP}$ & 0,95 & 17,8 \\
\hline $\mathrm{S}$ & $\log y=-2,09771+2,157904 * \log \mathrm{DAP}$ & 0,90 & 20,1 \\
\hline \multicolumn{4}{|c|}{ Casca } \\
\hline $\mathrm{N}$ & $\log \mathrm{y}=-0,699814+2,267223 * \log \mathrm{DAP}$ & 0,96 & 5,7 \\
\hline $\mathrm{P}$ & $\log y=-2,38515+2,494811 * \log \mathrm{DAP}$ & 0,95 & 12,9 \\
\hline $\mathrm{K}$ & $\log y=-0,90684+2,213217 * \log \mathrm{DAP}$ & 0,95 & 8,0 \\
\hline $\mathrm{Ca}$ & $\log y=-0,96013+2,349763 * \log \mathrm{DAP}$ & 0,89 & 12,0 \\
\hline $\mathrm{Mg}$ & $\log y=-1,08914+1,887263 * \log$ DAP & 0,85 & 20,1 \\
\hline $\mathrm{S}$ & $\log y=-2,57381+2,530617 * \log \mathrm{DAP}$ & 0,90 & 28,7 \\
\hline \multicolumn{4}{|c|}{ Folha } \\
\hline $\mathrm{N}$ & $\log \mathrm{y}=-1,604550+3,048978 * \log \mathrm{DAP}$ & 0,85 & 17,9 \\
\hline $\mathrm{P}$ & $\log y=-2,845660+2,901848 * \log$ DAP & 0,84 & 30,2 \\
\hline $\mathrm{K}$ & $\log y=-2,015770+2,986838 * \log$ DAP & 0,84 & 26,1 \\
\hline $\mathrm{Ca}$ & $\log y=-2,200940+2,952921 * \log$ DAP & 0,82 & 35,8 \\
\hline $\mathrm{Mg}$ & $\log y=-2,496890+2,205139 * \log \mathrm{DAP}$ & 0,84 & 24,5 \\
\hline $\mathrm{S}$ & $\log y=-3,234730+3,109386 * \log$ DAP & 0,87 & 27,0 \\
\hline \multicolumn{4}{|c|}{ Madeira } \\
\hline $\mathrm{N}$ & $\log y=-1,32089+2,805139 * \log \mathrm{DAP}$ & 0,99 & 3,8 \\
\hline $\mathrm{P}$ & $\log y=-2,51853+2,692839 * \log \mathrm{DAP}$ & 0,89 & 22,7 \\
\hline $\mathrm{K}$ & $\log \mathrm{y}=-1,0556+2,443427 * \log \mathrm{DAP}$ & 0,97 & 5,7 \\
\hline $\mathrm{Ca}$ & $\log y=-1,55988+3,180304 * \log \mathrm{DAP}$ & 0,80 & 19,9 \\
\hline $\mathrm{Mg}$ & $\log y=-1,85390+2,786079 * \log \mathrm{DAP}$ & 0,95 & 13,8 \\
\hline $\mathrm{S}$ & $\log y=-1,82693+2,371526 * \log \mathrm{DAP}$ & 0,91 & 25,9 \\
\hline \multicolumn{4}{|c|}{ Galho morto } \\
\hline $\mathrm{N}$ & $\log y=-1,07534+2,565744 * \log \mathrm{DAP}$ & 0,95 & 7,9 \\
\hline $\mathrm{P}$ & $\log y=-2,39851+2,381968 * \log \mathrm{DAP}$ & 0,92 & 12,3 \\
\hline $\mathrm{K}$ & $\log y=-1,29927+2,248503 * \log \mathrm{DAP}$ & 0,87 & 18,3 \\
\hline $\mathrm{Ca}$ & $\log y=-1,30667+2,608972 * \log \mathrm{DAP}$ & 0,94 & 10,1 \\
\hline $\mathrm{Mg}$ & $\log y=-1,83566+2,683534 * \log \mathrm{DAP}$ & 0,94 & 15,6 \\
\hline $\mathrm{S}$ & $\log y=-2,07172+2,219842 * \log \mathrm{DAP}$ & 0,87 & 16,2 \\
\hline \multicolumn{4}{|c|}{ Galho vivo } \\
\hline $\mathrm{N}$ & $\log y=-0,98270+2,538917 * \log$ DAP & 0,95 & 7,7 \\
\hline $\mathrm{P}$ & $\log y=-1,91438+2,227334 * \log \mathrm{DAP}$ & 0,91 & 43,8 \\
\hline $\mathrm{K}$ & $\log y=-0,84445+2,300289 * \log \mathrm{DAP}$ & 0,93 & 9,1 \\
\hline $\mathrm{Ca}$ & $\log y=-1,18243+2,358048 * \log \mathrm{DAP}$ & 0,92 & 11,7 \\
\hline $\mathrm{Mg}$ & $\log y=-2,14289+2,851488 * \log \mathrm{DAP}$ & 0,93 & 21,8 \\
\hline $\mathrm{S}$ & $\log y=-2,16490+2,328748 * \log$ DAP & 0,92 & 13,7 \\
\hline
\end{tabular}

$\mathrm{R}_{\mathrm{aj}}{ }_{\mathrm{aj}}$ coeficiente de determinação; $\mathrm{S}_{\mathrm{yx}}(\%)$ : erro padrão. O erro padrão da estimativa foi calculado para log $\mathrm{y}$.

Andrae; Krapfenbauer (1983a) verificaram que N, P, K e Mg, tanto para madeira quanto para casca, em araucária com 17 anos, apresentaram aumento das concentrações com aumento da altura relativa, ratificando a influência da idade dos tecidos nas concentrações nas diferentes alturas. $\mathrm{O}$ mesmo 
não foi apresentado pelo $\mathrm{Ca}$, o qual, devido a sua pequena mobilidade natural, acumulou-se nos tecidos mais velhos, principalmente nos da casca.

Tabela 6. Quantidade de macronutrientes $\left(\mathrm{kg} \cdot \mathrm{ha}^{-1}\right)$ encontrada na biomassa, por hectare, dos diferentes componentes das árvores de Acacia mearnsii De Wild. com quatro anos de idade, Arroio dos Ratos, RS/Brasil.

Table 6. Macronutrients quantity $\left(\mathrm{kg}_{\mathrm{g}} \mathrm{ha}^{-1}\right)$ found in different components biomass, per hectare, of four years old Acacia mearnsii De Wild. trees, Arroio dos Ratos, RS/Brazil.

\begin{tabular}{lccccccc}
\hline Componente & $\mathbf{N}$ & $\mathbf{P}$ & $\mathbf{K}$ & $\mathbf{C a}$ & $\mathbf{M g}$ & $\mathbf{S}$ & Total \\
\hline Folha & 69,11 & 2,84 & 23,24 & 14,08 & 5,94 & 1,87 & 117,08 \\
Raiz & 47,91 & 1,79 & 21,68 & 22,74 & 4,22 & 2,76 & 101,10 \\
Madeira & 73,66 & 4,20 & 60,60 & 133,79 & 20,98 & 8,39 & 301,62 \\
Casca & 75,32 & 2,62 & 42,20 & 55,18 & 12,91 & 1,91 & 190,14 \\
Exportação de micronutrientes (madeira & 43 & 44 & 51 & 71 & 58 & 55 & - \\
+ casca) em percentagem & 29,26 & 0,94 & 8,98 & 19,10 & 6,70 & 1,41 & 66,39 \\
Galho morto & 53,69 & 3,23 & 43,35 & 22,84 & 7,79 & 2,23 & 133,13 \\
Galho vivo & 348,95 & 15,62 & 200,05 & 267,73 & 58,54 & 18,57 & 909,46 \\
\hline Total & & & & & & & \\
\hline
\end{tabular}

Tabela 7. Teores médios de macronutrientes $\left(\mathrm{g}^{\mathrm{kg}} \mathrm{kg}^{-1}\right)$ ao longo do fuste para os componentes madeira e casca de Acacia mearnsii De Wild. com quatro anos de idade, Arroio dos Ratos, RS/Brasil.

Table 7. Macronutrients average levels (g.kg $)$ along the trunk for wood and bark components of four years old Acacia mearnsii De Wild. trees, Arroio dos Ratos, RS/Brazil.

\begin{tabular}{lcccccc}
\hline \% da altura total & $\mathbf{N}$ & $\mathbf{P}$ & $\mathbf{K}$ & $\mathbf{C a}$ & $\mathbf{M g}$ & $\mathbf{S}$ \\
\hline 1 & & & \multicolumn{2}{c}{ Madeira } & & \\
3 & $1,44 \mathrm{c}^{*}$ & $0,09 \mathrm{a}$ & $1,37 \mathrm{ab}$ & $3,65 \mathrm{a}$ & $0,50 \mathrm{a}$ & $0,19 \mathrm{a}$ \\
10 & $1,34 \mathrm{c}$ & $0,07 \mathrm{a}$ & $1,11 \mathrm{ab}$ & $3,31 \mathrm{a}$ & $0,53 \mathrm{a}$ & $0,19 \mathrm{a}$ \\
20 & $1,32 \mathrm{c}$ & $0,06 \mathrm{a}$ & $0,96 \mathrm{~b}$ & $3,07 \mathrm{a}$ & $0,48 \mathrm{a}$ & $0,15 \mathrm{a}$ \\
30 & $1,33 \mathrm{c}$ & $0,06 \mathrm{a}$ & $0,95 \mathrm{~b}$ & $3,11 \mathrm{a}$ & $0,41 \mathrm{a}$ & $0,18 \mathrm{a}$ \\
40 & $1,38 \mathrm{c}$ & $0,07 \mathrm{a}$ & $1,05 \mathrm{~b}$ & $3,26 \mathrm{a}$ & $0,46 \mathrm{a}$ & $0,19 \mathrm{a}$ \\
50 & $1,37 \mathrm{c}$ & $0,09 \mathrm{a}$ & $1,26 \mathrm{ab}$ & $2,54 \mathrm{a}$ & $0,40 \mathrm{a}$ & $0,17 \mathrm{a}$ \\
60 & $1,51 \mathrm{bc}$ & $0,09 \mathrm{a}$ & $1,34 \mathrm{ab}$ & $3,04 \mathrm{a}$ & $0,46 \mathrm{a}$ & $0,19 \mathrm{a}$ \\
70 & $1,61 \mathrm{abc}$ & $0,08 \mathrm{a}$ & $1,19 \mathrm{ab}$ & $3,00 \mathrm{a}$ & $0,41 \mathrm{a}$ & $0,16 \mathrm{a}$ \\
80 & $1,76 \mathrm{abc}$ & $0,08 \mathrm{a}$ & $1,44 \mathrm{ab}$ & $2,25 \mathrm{a}$ & $0,41 \mathrm{a}$ & $0,19 \mathrm{a}$ \\
90 & $1,94 \mathrm{ab}$ & $0,07 \mathrm{a}$ & $1,71 \mathrm{a}$ & $2,25 \mathrm{a}$ & $0,41 \mathrm{a}$ & $0,17 \mathrm{a}$ \\
\hline & $2,05 \mathrm{a}$ & $0,11 \mathrm{a}$ & $1,55 \mathrm{ab}$ & $2,94 \mathrm{a}$ & $0,45 \mathrm{a}$ & $0,20 \mathrm{a}$ \\
\hline 1 & & & & $C a s c a$ & & \\
3 & $9,59 \mathrm{~b}$ & $0,37 \mathrm{bcd}$ & $6,11 \mathrm{a}$ & $9,08 \mathrm{a}$ & $1,44 \mathrm{a}$ & $0,27 \mathrm{a}$ \\
10 & $9,40 \mathrm{~b}$ & $0,34 \mathrm{~d}$ & $5,46 \mathrm{a}$ & $8,75 \mathrm{a}$ & $1,44 \mathrm{a}$ & $0,22 \mathrm{a}$ \\
20 & $9,43 \mathrm{~b}$ & $0,35 \mathrm{~d}$ & $5,62 \mathrm{a}$ & $7,61 \mathrm{a}$ & $1,52 \mathrm{a}$ & $0,27 \mathrm{a}$ \\
30 & $10,10 \mathrm{ab}$ & $0,36 \mathrm{~cd}$ & $5,82 \mathrm{a}$ & $8,58 \mathrm{a}$ & $1,57 \mathrm{a}$ & $0,25 \mathrm{a}$ \\
40 & $10,36 \mathrm{ab}$ & $0,35 \mathrm{~d}$ & $5,48 \mathrm{a}$ & $7,16 \mathrm{a}$ & $1,52 \mathrm{a}$ & $0,30 \mathrm{a}$ \\
50 & $10,79 \mathrm{ab}$ & $0,37 \mathrm{bcd}$ & $5,92 \mathrm{a}$ & $7,27 \mathrm{a}$ & $1,67 \mathrm{a}$ & $0,28 \mathrm{a}$ \\
60 & $11,19 \mathrm{ab}$ & $0,40 \mathrm{abcd}$ & $5,80 \mathrm{a}$ & $6,43 \mathrm{a}$ & $1,69 \mathrm{a}$ & $0,32 \mathrm{a}$ \\
70 & $11,06 \mathrm{ab}$ & $0,42 \mathrm{abcd}$ & $5,80 \mathrm{a}$ & $7,14 \mathrm{a}$ & $2,82 \mathrm{a}$ & $0,25 \mathrm{a}$ \\
80 & $10,70 \mathrm{ab}$ & $0,45 \mathrm{a}$ & $5,21 \mathrm{a}$ & $6,25 \mathrm{a}$ & $1,85 \mathrm{a}$ & $0,26 \mathrm{a}$ \\
90 & $11,78 \mathrm{a}$ & $0,44 \mathrm{ab}$ & $6,37 \mathrm{a}$ & $6,78 \mathrm{a}$ & $2,04 \mathrm{a}$ & $0,31 \mathrm{a}$ \\
\hline
\end{tabular}

*Médias seguidas pela mesma letra na vertical não diferem pelo Teste de Tukey a 5\% de probabilidade.

\section{Teor representativo}

A tabela 8 mostra os valores do somatório da massa de nutrientes, do somatório da biomassa total amostrada e dos teores representativos dos macronutrientes ao longo do fuste para os componentes madeira e casca de acácia-negra. Tanto para madeira como para casca, quanto maior for a massa total de 
macronutrientes maior será o teor representativo, principalmente do $\mathrm{N}$ e Ca. Os teores de Ca mais elevados na casca são devido ao Ca estar associado à parede celular, não sendo redistribuído, ocorrendo a diminuição do seu teor com o desenvolvimento da planta (LAMBERS et al., 2000).

Tabela 8. Valores do somatório da massa de nutrientes e da biomassa e dos teores representativos para macronutrientes no fuste, para os componentes madeira e casca de Acacia mearnsii De Wild. com quatro anos de idade, Arroio dos Ratos, RS/Brasil.

Table 8. Accumulated values of nutrients mass and biomass and representative levels of macronutrients in the trunk, for wood and bark components of four years old Acacia mearnsii De Wild. trees, Arroio dos Ratos, RS/Brazil.

\begin{tabular}{lcccccc}
\hline \multirow{2}{*}{ Macronutriente } & \multicolumn{3}{c}{ Madeira } & \multicolumn{3}{c}{ Casca } \\
\cline { 2 - 7 } & Massa $(\mathbf{g})$ & Biomassa $(\mathbf{k g})$ & $\mathbf{T R}^{\mathbf{1}}\left(\mathbf{g} \cdot \mathbf{k g}^{-\mathbf{1}}\right)$ & Massa $(\mathbf{g})$ & Biomassa $(\mathbf{k g})$ & $\mathbf{T R}^{\mathbf{1}}\left(\mathbf{g . k g}^{-\mathbf{1}}\right)$ \\
\hline $\mathrm{N}$ & $2.021,90$ & $1.351,00$ & 1,50 & 1971,24 & 194,11 & 10,16 \\
$\mathrm{P}$ & 110,53 & $1.351,00$ & 0,08 & 78,81 & 194,11 & 0,41 \\
$\mathrm{~K}$ & $1.742,79$ & $1.351,00$ & 1,29 & $1.048,58$ & 194,11 & 5,40 \\
$\mathrm{Ca}$ & $4.241,72$ & $1.351,00$ & 3,14 & $1.383,4$ & 194,11 & 7,13 \\
$\mathrm{Mg}$ & 607,94 & $1.351,00$ & 0,45 & 305,25 & 194,11 & 1,57 \\
$\mathrm{~S}$ & 245,5 & $1.351,00$ & 0,14 & 54,56 & 194,11 & 0,28 \\
\hline
\end{tabular}

${ }^{1}$ Teor representativo em g. $\mathrm{kg}^{-1}$ para macronutrientes; Biomassa: biomassa total do fuste, para madeira e casca, das 21 árvores amostradas; Massa: massa total de nutrientes das 21 árvores no fuste para madeira e casca.

\section{Altura representativa}

A tabela 9 mostra a variação da altura representativa com base nos diferentes teores dos macronutrientes ao longo do fuste e os teores representativos calculados. Verificou-se que, para os elementos que apresentaram variação dos teores ao longo do fuste, $\mathrm{N}$ na madeira é o único macronutriente em que a altura para a amostragem foi de $50 \%$ da altura total das árvores de acácia-negra. No entanto, para $\mathrm{N}$ e $\mathrm{P}$ na casca, a altura média foi, respectivamente, de 45 e $55 \%$. No caso do K, na madeira, a altura para a amostragem foi de $55 \%$.

Tabela 9. Altura representativa (dada em percentagem da altura total), que representa o ponto de amostragem dos macronutrientes nos componentes (madeira e casca) no fuste de Acacia mearnsii De Wild. com quatro anos de idade, Arroio dos Ratos, RS/Brasil.

Table 9. Representative height (in total height percentage), that represents the sampling point of macronutrients in wood and bark components of four years old Acacia mearnsii De Wild. trunk, Arroio dos Ratos, RS/Brazil.

\begin{tabular}{lccccccc}
\hline Componente & Variável & $\mathbf{N}$ & $\mathbf{P}$ & $\mathbf{K}$ & $\mathbf{C a}$ & $\mathbf{M g}$ & $\mathbf{S}$ \\
\hline \multirow{2}{*}{ Madeira } & $\mathrm{TR}^{1}$ & 1,50 & 0,08 & 1,29 & 3,14 & 0,45 & 0,14 \\
& $\mathrm{AR}^{2}$ & $50 \%$ & $1-100 \%$ & $40-70 \%$ & $1-100 \%$ & $1-100 \%$ & $1-100 \%$ \\
\hline \multirow{2}{*}{ Casca } & $\mathrm{TR}$ & 10,16 & 0,41 & 5,40 & 7,13 & 1,57 & 0,28 \\
& $\mathrm{AR}$ & $20-70 \%$ & $50-60 \%$ & $1-100 \%$ & $1-100 \%$ & $1-100 \%$ & $1-100 \%$ \\
\hline \multirow{2}{*}{$\mathrm{TR}^{1}:$ teor representativo, $\mathrm{AR}^{2}:$ altura representativa. } & & & & &
\end{tabular}

Comparando os dados da tabela 9 com os trabalhos de Andrae e Krapfenbauer (1983b) e Hoppe (2003), é possível analisar que os dados não coincidem com os dados do presente trabalho. Hoppe (2003) determinou, com base na análise da distribuição dos teores de macronutrientes ao longo do fuste, que o ponto de coleta para amostras dos teores dos macronutrientes, no componente madeira e casca, deve ser localizado a 26,2\% da altura total das árvores de Platanus $x$ acerifolia. Andrae; Krapfenbauer (1983b) determinaram que para Eucalyptus saligna a altura do ponto de amostragem para o teor dos nutrientes fica em torno de $23 \%$ da altura total.

Essa diferença entre os dados do presente estudo com os dados de Andrae; Krapfenbauer (1983b) e Hoppe (2003) pode ser em função de que os teores de nutrientes na casca e na madeira podem apresentar variações com a idade e o tamanho das árvores, pois árvores menores tendem a apresentar maiores teores (CLEVELARIO JÚNIOR, 1996).

Os teores de nutrientes na biomassa são em função de vários fatores, entre eles, pragas e enfermidades, idade das árvores e das folhas, posição na copa, estação do ano, qualidade do sítio, 
procedência, variação conforme o elemento, características nutricionais de cada espécie, disponibilidade de nutrientes no solo, componente a ser analisado (BINKLEY, 1986; PRITCHETT, 1990; BELLOTE; SILVA, 2000; CALDEIRA, 2003), bem como as características genéticas pertinentes a cada espécie (SCHUMACHER, 1995; CALDEIRA, 2003).

Dos fatores citados que influenciam o teor de nutrientes na biomassa das árvores, dois merecem destaque: a idade do material amostrado e a espécie, pois, de acordo com Bellote e Silva (2000), a idade do material amostrado se refere à idade fisiológica das folhas, em que o teor de nutrientes nesses órgãos depende, principalmente, do estádio fisiológico e da oferta de nutrientes no solo.

Cabe ressaltar que os dados do presente estudo (Tabela 9) são similares aos dados encontrados por Caldeira (1998) e Barichello (2003) para Acacia mearnsii com 2,4 e 8 anos, respectivamente, e Weber (2004) para um povoamento de Cryptomeria japonica com 28 anos, que utilizaram 50\% da altura total para retirada da amostra do fuste (casca + madeira) e determinação do teor de nutrientes nesses componentes, bem como para determinação do teor de umidade, para cálculo da biomassa seca.

\section{CONCLUSÕES}

No ambiente onde foi desenvolvida a presente pesquisa com de Acacia mearnsii com quatro anos de idade, conclui-se que:

- De todos os macronutrientes analisados, apenas $\mathrm{N}$ e $\mathrm{P}$ na casca e $\mathrm{K}$ na madeira apresentaram diferenças nos teores ao longo do fuste.

- Para quantificar os macronutrientes no fuste (madeira e casca), o ponto ótimo de amostragem deve ser a 50\% da altura total das árvores.

- É possível estimar com segurança o estoque de macronutrientes por compartimento das árvores de Acacia mearnsii com o uso de equações alométricas a partir do DAP.

\section{REFERÊNCIAS}

ANDRAE, F. H.; KRAPFENBAUER, A. Estudos da situação da biomassa e de nutrientes de um reflorestamento de quatro anos com Eucalyptus saligna Smith em Santa Maria, RS. In: ANDRAE, F. H.; KRAPFENBAUER, A. (Eds). Pesquisa Austro-Brasileira 1973-1982 sobre Araucaria angustifolia, Podocarpus lambertii e Eucalyptus saligna. Santa Maria-Brasil: Universidade Federal de Santa Maria, 1983b. p. 68-85.

ANDRAE, F. H.; KRAPFENBAUER, A. Inventário de um reflorestamento de Araucaria de 17 anos em Passo Fundo, RS. Parte II: Inventário de nutrientes. In: ANDRAE, F. H.; KRAPFENBAUER, A. (Eds). Pesquisa Austro-Brasileira 1973-1982 sobre Araucaria angustifolia, Podocarpus lambertii e Eucalyptus saligna. Santa Maria-Brasil: Universidade Federal de Santa Maria, 1983a, p. 30-55.

BARICHELLO, L. Quantificação da biomassa e dos nutrientes em floresta de Acacia mearnii De Wild. na região sul do Brasil. 58 f. Dissertação (Mestrado em Engenharia Florestal) - Universidade Federal de Santa Maria, Santa Maria, 2003.

BELlOTE, A. F. J. Suprimento de nutrientes minerais e crescimento de plantações adubadas de Eucaliptus grandis nos cerrados do Estado de São Paulo. 166 f. Tese (Doutorado) - Universidade de Fraiburg, Fraiburg, 1990.

BELLOTE, A. F. J.; SILVA, H. D. Técnicas de amostragem e avaliações nutricionais em plantios de Eucalyptus spp. In: GONÇALVES, J. L. M.; BENEDETTI, V. (Eds.) Nutrição e fertilização florestal. Piracicaba: ESALQ/USP, 2000. p. 105-133.

BINKLEY, D. Forest nutrition management. New York: J. Wiley \& Sons, 1986. 290 p.

CALDEIRA, M. V. W. Determinação de biomassa e nutrientes em uma Floresta Ombrófila Mista Montana em General Carneiro, Paraná.. 176f. Tese (Doutorado em Ciências Florestais) - Setor de Ciências Agrárias, Universidade Federal do Paraná, Curitiba, 2003 
CALDEIRA, M. V. W. Quantificação da biomassa e do conteúdo de nutrientes em diferentes procedências de Acácia-negra (Acacia mearnsii De wild.). 96f. Dissertação (Mestrado em Engenharia Florestal) - Universidade Federal de Santa Maria, Santa Maria, 1998.

CALDEIRA, M. V. W.; SCHUMACHER, M. V.; SCHEEREN, L. W.; BARICHELLO, L. R.; WATZLAWICK, L. F. Relação hipsométrica para Acacia mearnsii com diferentes idades. Boletim de Pesquisa Florestal, Colombo, PR, n. 45, p. 57-68, 2002.

CALIL, F. N. Aspectos da ciclagem de nutrientes em um sistema silvopastoril com Acacia mearnsii De Wild., no município de Tupanciretã, RS. 77 f. Dissertação (Mestrado em Engenharia Florestal) Universidade Federal de Santa Maria, Santa Maria, 2003.

CLEVELARIO JÚNIOR, J. Distribuição de carbono e de elementos minerais em um ecossistema florestal tropical úmido baixo-montano. $135 \mathrm{f}$. Tese (Doutorado em Solos e Nutrição de Plantas) Universidade Federal de Viçosa, Viçosa, MG. 1996.

EMPRESA BRASILEIRA DE PESQUISA AGROPECUÁRIA (EMBRAPA). Sistema brasileiro de classificação e levantamento de solos. Rio de Janeiro, 1999. 412 p.

FINGER, C. A. G. Fundamentos de biometria florestal. Santa Maria: UFSM/CEPEF / FATEC, 1992. $201 \mathrm{p}$.

FRANCO, A. A.; CAMPELLO, E. F.; DIAS, L. E.; FARIA, S. M. Revegetação de áreas de mineração de bauxita em Porto Trombetas - PA com leguminosas arbóreas noduladas e micorrizadas. In: SIMPÓSIO SUL-AMERICANO, 1.; SIMPÓSIO NACIONAL DE RECUPERAÇÃO DE ÁREAS DEGRADADAS, 2., 1994, Foz do Iguaçu. Anais... Curitiba: FUPEF, 1994, p. 145-153.

FREITAS, R. do A. Estudo da biomassa e do conteúdo de nutrientes em um povoamento de Eucalyptus grandis Hil ex Maiden plantado em solo sujeito à arenização no município de AlegreteRS. 60 f. Dissertação (Mestrado em Engenharia Florestal) - Universidade Federal de Santa Maria, Santa Maria, 2000.

GONÇALVES, J. L. M.; STAPE, J. L.; BENEDETTI, V.; FESSEL, V. A. G.; GAVA, J. L. Reflexos do cultivo mínimo e intensivo do solo em sua fertilidade e na nutrição das árvores. In: GONÇALVES, J.L.M.; BENEDETTI, V. (Eds.) Nutrição e fertilização florestal. Piracicaba: ESALQ/USP. 2000. p. 357.

HOPPE, J. M. Biomassa e nutrientes em Platanus $x$ acerifolia (Aiton) Willd. estabelecido no município de Dom Feliciano-RS. 143 f. Tese (Doutorado em Engenharia Florestal) - Universidade Federal de Santa Maria, Santa Maria, 2003.

INSTITUTO DE PESQUISAS AGRONÔMICAS (IPAGRO). Atlas agroclimático do Estado do Rio Grande do Sul. Porto Alegre, 1989.: 3 v.

INVENTÁRIO FLORESTAL CONTÍNUO DO RS. Disponível em: <http://coralx.ufsm.br/ifcrs/conclu.htm> Acesso em: 26/05/2005.

KRAMER, R. J.; KOZLOWSKI, T. T. Fisiologia das árvores. Lisboa: Fundação Kalouste Goulbenkian, $1972,745 \mathrm{p}$.

LAMBERS, H.; CHAPIN III, F. S.; PONS, T. L. Plant physiological ecology. New York: SpringerVerlag, 2000. $540 \mathrm{p}$.

MORENO, J. A. Clima do Rio Grande do Sul. Porto Alegre: Secretaria da Agricultura, 1961, 42 p.

PEREIRA, J. C.; CALDEIRA, M. V. W.; SCHUMACHER, M. V.; HOPPE, J. M.; SANTOS, E. M. Estimativa do conteúdo de nutrientes em um povoamento de Acacia mearnsii De Wild. no Rio Grande do Sul - Brasil. Revista Árvore, Viçosa, MG, v. 24, n. 2, p. 193-199, 2000.

POGGIANI, F.; ZEN, S.; MENDES, F.S.; SPINA-FRANÇA, F. Ciclagem e exportação de nutrientes em florestas para fins energéticos. IPEF, Piracicaba, n. 27, p. 17-30, 1984. 
PRITCHETT, W. L. Suelos forestales: Propriedades, conservacíon mejoramiento. 2 ed. México: Limusa Noriega, 1990. $634 \mathrm{p}$.

SAIDELLES, F. L. F. Determinação da biomassa e altura de amostragem para a quantificação de nutrientes em Acacia mearnsii De Wild. 97 f. Tese (Doutorado em Engenharia Florestal) - Universidade Federal de Santa Maria, Santa Maria, 2005.

SCHENEIDER, P. R.; HOSOKAWA, R. T. Estudo de equações volumétricas para tabelas de volume com e sem casca para Acácia Negra (Acacia mearnsii De Wild). In: CONGRESSO FLORESTAL BRASILEIRO, 3., 1978, Manaus. Anais... Manaus: Sociedade Brasileira de Silvicultura, 1978, p. 90-95.

SCHUMACHER, M. V. Naehrstoffkreislauf in verschiedenen Bestaeden von Eucalyptus saligna (Smith), Eucalyptus dunnii (Maiden) und Eucalyptus globulus (Labillardière) in Rio Grande do Sul, Brasilien. 167 f. Tese (Doutorado em Ecologia e Nutrição Florestal) - Universitäet für Bodenkultur, Wien, 1995.

SETA. Disponível em: <http://www.seta-sa.com.br/>. Acesso em: 13/01/2005.

TEDESCO, M. J.; GIANELlO, C.; BISSANI, C. A.; BOHNEN, H.; VOLKWEISS, S. J. Análises de solos, plantas e outros materiais. 2.ed. Porto Alegre: UFRGS, 1995. (Boletim Técnico, n. 5).

VAN DER DRIESSCHE, R. Prediction of mineral status of trees by foliar analysis. The Botanical Review. New York, v. 40, p. 347-394, 1984.

WEBER, C. Biomassa e nutrientes em um povoamento de Cryptomeria japonica (L. F.) D. Don., no município de Encruzilhada do Sul-Rio Grande do Sul. Santa Maria: Universidade Federal de Santa Maria, 2004. 36 f. Relatório de Estágio Supervisionado em Engenharia Florestal. 
FLORESTA, Curitiba, PR, v. 40, n. 1, p. 49-62, jan./mar. 2010. Saidelles, F. L. F. et al. 